Pacific

Journal of

Mathematics

ON THE GEOMETRY OF TWO DIMENSIONAL PRYM VARIETIES

\author{
R. Smith and R. VARLey
}




\title{
ON THE GEOMETRY OF TWO DIMENSIONAL PRYM VARIETIES
}

\author{
R. SMith AND R. VARLEy
}

If $\Sigma$ is a smooth genus two curve, $\Sigma \subset \operatorname{Pic}^{1}(\Sigma)$ the Abel embedding in the degree one Picard variety, $|2 \Sigma|$ the projective space parametrizing divisors on $\operatorname{Pic}^{1}(\Sigma)$ linearly equivalent to $2 \Sigma$, and $\operatorname{Pic}^{0}(\Sigma)_{2}=G \cong(\mathbb{Z} / 2 \mathbb{Z})^{4}$ the subgroup of points of order two in the Jacobian variety $J(\Sigma)=\operatorname{Pic}^{0}(\Sigma)$, then $G$ acts on $|2 \Sigma|$ and the quotient variety $|2 \Sigma| / G$ parametrizes two fundamental moduli spaces associated with the curve $\Sigma$. Namely, Narasimhan-Ramanan's work implies an isomorphism of $|2 \Sigma| / G$ with the space $\mathcal{M}$ of ( $S$-equivalence classes of semistable, even) $\mathbb{P}^{1}$ bundles over $\Sigma$, and Verra has defined a precise birational correspondence between $|2 \Sigma| / G$ and Beauville's compactification of $\mathcal{P}^{-1}(J(\Sigma))$ the fiber of the classical Prym map over $J(\Sigma)$. In this paper we give a new (birational) construction of the composed Narasimhan-Ramanan-Verra map $\alpha: \mathcal{M} \rightarrow \mathcal{P}^{-1}(J(\Sigma))$, defined purely in terms of the geometry of a (generic stable) $\mathbb{P}^{1}$ bundle $X \rightarrow \Sigma$ in $\mathcal{M}$, and also an explicit rational inverse map $\beta: \mathcal{P}^{-1}(J(\Sigma)) \rightarrow \mathcal{M}$. The map $\alpha$ may be viewed as an analog for Prym varieties of Andreotti's reconstruction of a curve $C$ of genus $g$ from the branch locus of the canonical map on the symmetric product $C^{(g-1)}$. The map $\beta$ assigns to an étale double cover $\pi: \tilde{C} \rightarrow C$ in $\mathcal{P}^{-1}(J(\Sigma))$, where $\tilde{C}$ and $C$ are curves of genera 5 and 3 respectively, the $\mathbb{P}^{1}$ bundle $\varphi: X \rightarrow \Sigma$, where $X=$ \{divisors $D$ in $\tilde{C}^{(4)}: \pi_{*}(D) \equiv \omega_{C}$, and $h^{0}(D)$ is even\} and $\varphi: X \rightarrow \varphi(X) \cong \Sigma \subset \operatorname{Pic}^{4}(\tilde{C})$ is the Abel map.

\section{Introduction.}

To motivate the constructions in this paper, recall that Torelli's problem for a curve $C$ usually means recovering $C$ from the theta divisor $\Theta$ of the Jacobian variety $J(C)$. Andreotti showed it is equivalent to recover $C$ from its symmetric product $C^{(g-1)}$ and, by pulling back the canonical line bundle $K_{\Theta}$ via the Abel map $C^{(g-1)} \rightarrow \Theta$, he recovered $C$ from the branch locus of the associated canonical map $C^{(g-1)} \rightarrow \mathbb{P}^{g-1}([\mathbf{A}])$. In the case of a Prym variety of a double cover of curves $\pi: \tilde{C} \rightarrow C$, the theta divisor $\Xi$ of the Prym variety $P(\tilde{C} / C)$ does not always determine the double cover. In particular a 
double cover of a curve $C$ of genus three depends on 6 parameters while the theta divisor of the two dimensional Prym variety $(P, \Xi)$ depends on only 3 . Hence the Prym theta divisor $\Xi$ cannot determine uniquely the double cover $\pi: \tilde{C} \rightarrow C$, (although a prescription can be given by which $\Xi$ determines all double covers with Prym variety $P$ ). If one takes the analog of Andreotti's approach however, starting from the Abel parametrization of the Prym theta divisor $\tilde{C}^{(2 g-2)} \supset X \rightarrow \Xi$, (see Section 2 for the precise definition), it turns out there is sometimes more information in $X$ than is contained in $\Xi$. The key is that the map $\varphi: X \rightarrow \Xi$ is not birational, but (generically) a $\mathbb{P}^{1}$ bundle. This is the approach taken by H. Yin in $[\mathbf{Y}]$, where he proves the following infinitesimal Torelli result: If $C$ is nonhyperelliptic of genus three, then $\tilde{C}$ is birational to a component of the Hilbert scheme of sections of the $\mathbb{P}^{1}$ bundle $X \rightarrow \Xi$, and $X$ has the same number of moduli as does the double cover $\pi: \tilde{C} \rightarrow C$, i.e. $T^{1}(\tilde{C} / C) \cong T^{1}(X)$. This means essentially that the map from the space of double covers $\tilde{C} / C$ to the space of all $X$ 's is a local isomorphism. In the present article we extend the results of Yin and the parallel with Andreotti's work, and link them with the results of Narasimhan-Ramanan and of Verra, on rank 2 vector bundles and the fibers of the Prym map over two dimensional abelian varieties. In particular we show (when $g(C)=3$ and $C$ is smooth, nonhyperelliptic), that $X$ determines $\tilde{C} / C$ uniquely, and that the correspondence between $X$ and $\tilde{C} / C$ is induced by Narasimhan-Ramanan's equivalence between semi-stable vector bundles on $\Xi$ and divisors in $|2 \Theta|$ on $\operatorname{Pic}^{1}(\Xi)$. We discuss this in more detail next.

i) In $[\mathbf{N}-\mathbf{R}]$ Narasimhan and Ramanan give an isomorphism from the set $\mathcal{M}$ of $S$-equivalence classes of semi-stable rank 2 vector bundles with determinant $\mathcal{O}$ on a genus two curve $\Sigma$, to the three dimensional linear system $|2 \Theta|$ on $\operatorname{Pic}^{1}(\Sigma)$, by associating to each rank 2 bundle $\mathcal{E}$ the set $D_{\mathcal{E}}$ of invertible sheaves $\xi$ in $\operatorname{Pic}^{1}(\Sigma)$ such that $H^{0}(\mathcal{E} \otimes \xi) \neq 0$. They prove $D_{\mathcal{E}}$ is the support of a unique divisor $\tilde{C}_{\mathcal{E}}$ in $|2 \Theta|$, (where $\Theta=\Sigma \subset \operatorname{Pic}^{1}(\Sigma)$ is defined by the natural embedding $p \rightarrow \mathcal{O}(p)$ ), and that the map $\mathcal{M} \rightarrow|2 \Theta|$ taking $\mathcal{E}$ to $\tilde{C}_{\mathcal{E}}$ is bijective, (where a nonstable bundle $S$-equivalent to $\mu \oplus \mu^{-1}$ with $\mu$ in $\operatorname{Pic}^{0}(\Sigma)$ corresponds to the point $\Theta_{\mu}+\Theta_{\mu^{-1}}$ on the Kummer surface in $|2 \Theta|)$.

ii) With the same notation as above, Verra ([V]) describes the fibers of the extended Prym map $\mathcal{P}: \mathcal{R}_{3} \rightarrow \mathcal{A}_{2}$, [or $\bar{P}: \overline{\mathcal{R}}_{3} \rightarrow \mathcal{A}_{2}$ in Verra's notation], via a rational map $|2 \Theta| \rightarrow \mathcal{P}^{-1}(J(\Sigma))$. Since every divisor $\tilde{C}$ in $|2 \Theta|$ is invariant for the involution $\iota$ on $\operatorname{Pic}^{1}(\Sigma)$ taking $\xi$ to $K_{\Sigma}-\xi$, he defines this map by taking $\tilde{C}$ in $|2 \Theta|$ to $\tilde{C} \rightarrow C$ where $C=\tilde{C} / \iota$. He shows this map is defined except along the 16 conic "tropes" on the Kummer surface $\mathcal{K}$ in $|2 \Theta|$, and carries the complement $|2 \Theta|-\mathcal{K}$ onto the set of those irreducible stable double covers $(\tilde{C} / C)$ in $\mathcal{P}^{-1}(J(\Sigma))$ such that $\tilde{C}$ is not hyperelliptic. He shows also that if $\operatorname{Aut}(\Sigma) \cong \mathbb{Z}_{2}$, the fibers of the map $|2 \Theta| \rightarrow \mathcal{P}^{-1}(J(\Sigma))$ 
are orbits of the action on $|2 \Theta|$ by the group $G \cong\left(\mathbb{Z}_{2}\right)^{4}$ of points of order two in $\operatorname{Pic}^{0}(\Sigma)$. Finally he shows how to make the map $|2 \Theta| \rightarrow \mathcal{P}^{-1}(J(\Sigma))$ a surjective morphism by blowing up first at the 16 "points of order two" on the Kummer surface (the singular points on the union of the tropes), then along the strict transform of the tropes, so that the $G$ action on $|2 \Theta|$ extends and induces an isomorphism from the $G$-quotient of the blowup to $\mathcal{P}^{-1}(J(\Sigma))$. Moreover, the exceptional plane $\left|\mathcal{O}_{\Sigma}(2 \Sigma)\right|$ over a point of order two, the plane of bicanonical divisors on $\Sigma \cong \Theta$, parametrizes the hyperelliptic double covers $\tilde{C} \rightarrow C$ in $\mathcal{P}^{-1}(J(\Sigma))$, where each hyperelliptic curve $\tilde{C}$ arises as a double cover of $\Sigma$ branched over a bicanonical divisor. (The involution defining the double cover $\tilde{C} \rightarrow C$ is the product of the hyperelliptic involution on $\tilde{C}$ and the involution induced by the double cover $\tilde{C} \rightarrow \Sigma$.)

Since two vector bundles $\mathcal{E}, \tilde{\mathcal{E}}$ determine isomorphic $\mathbb{P}^{1}$ bundles if and only if $\mathcal{E} \cong \tilde{\mathcal{E}} \otimes \mathcal{L}$ for some line bundle $\mathcal{L}$, and since $\operatorname{det}(\mathcal{E} \otimes \mathcal{L})=\operatorname{det}(\mathcal{E}) \otimes \mathcal{L}^{2}$, two stable rank 2 vector bundles $\mathcal{E}, \tilde{\mathcal{E}}$ of determinant $\mathcal{O}$ on $\Sigma$ determine isomorphic $\mathbb{P}^{1}$ bundles if and only if $\mathcal{E} \cong \tilde{\mathcal{E}} \otimes \mathcal{L}$ where $\mathcal{L}^{2} \cong \mathcal{O}$. Moreover, this action of the group $G$ on $\mathcal{M}$ corresponds to the action on $|2 \Theta|$, so that stable $\mathbb{P}^{1}$ bundles on $\Sigma$ correspond via Narasimhan-Ramanan and Verra bijectively to the set of irreducible stable double covers $(\tilde{C} / C)$ in $\mathcal{P}^{-1}(J(\Sigma))$ such that $\tilde{C}$ is not hyperelliptic.

iii) In the present article we show that the $\mathbb{P}^{1}$ bundle $\varphi: X \rightarrow \Sigma$ associated by the Abel parametrization to a double cover $\tilde{C} \rightarrow C$ of a smooth nonhyperelliptic genus three curve with Prym variety $J(\Sigma)$ determines $\tilde{C} \rightarrow C$ uniquely, that $X$ arises from a stable rank 2 vector bundle on $\Sigma$ with determinant $\mathcal{O}$, and that the association $(\tilde{C} / C) \mapsto X$ inverts the maps of Narasimhan-Ramanan and Verra. Since we give an explicit inverse we also recover, at least for smooth nonhyperelliptic $\tilde{C}$, that their correspondence is bijective. We also reconstruct from $X$ a two parameter family of hyperelliptic double covers with the same Prym variety as $\tilde{C} / C$.

More precisely, by analogy with Andreotti's proof, we reconstruct the double cover $\pi: \tilde{C} \rightarrow C$, from the branch locus of the finite map $h: X \rightarrow \mathbb{P}^{2}$ associated to the line bundle $\mathcal{T}_{\varphi} \otimes \varphi^{*}\left(K_{\Sigma}\right)$, (the pulled back canonical bundle of the Prym theta divisor $\Xi \cong \Sigma$, twisted by the bundle $\mathcal{T}_{\varphi}$ of tangents "along the fibers" of $\varphi$ ). If $\tilde{C} \rightarrow C$ is an étale double cover of a smooth nonhyperelliptic genus three curve, with Prym variety $(P, \Xi) \cong(J(\Sigma), \Sigma)=$ the jacobian of a genus two curve $\Sigma$, we prove Yin's rank 2 vector bundle $\mathcal{E}$ on $\Sigma$ which defines the Abel $\mathbb{P}^{1}$ bundle $X \rightarrow \Sigma$, can be taken to have determinant $\mathcal{O}$ and is stable, and that the divisor $\tilde{C}_{\mathcal{E}}$ in $|2 \Theta|$ associated to $\mathcal{E}$ by Narasimhan-Ramanan is isomorphic to $\tilde{C}$. Conversely if $\mathcal{E}$ is any stable rank 2 vector bundle over $\Sigma$ with determinant $\mathcal{O}$, and if the corresponding divisor $\tilde{C}_{\mathcal{E}}=\tilde{C}$ in $|2 \Theta|$ is smooth, with natural involution $\xi \mapsto(K-\xi)$, we 
prove the associated Prym variety is isomorphic to $J(\Sigma)$, and the Abel $\mathbb{P}^{1}$ bundle $\varphi: X \rightarrow \Xi$ constructed from a Poincaré line bundle on $\operatorname{Pic}^{4}(\tilde{C}) \times \tilde{C}$, is isomorphic to $\mathbb{P}(\mathcal{E})$. This proves that the Prym construction inverts the Narasimhan-Ramanan correspondence at least for smooth divisors in $|2 \Theta|$, and hence recovers for these divisors that their correspondence is bijective. Finally we show that the fibers of the map $h: X \rightarrow \mathbb{P}^{2}$ over lines yield hyperelliptic double covers $\pi: \tilde{C}_{\lambda} \rightarrow C_{\lambda}$ with the same Prym variety as $\pi: \tilde{C} \rightarrow C$.

\section{Background: The Prym variety of a double cover $\pi: \tilde{C} \rightarrow C$.}

Let $\tilde{C} / C$ denote an étale double cover $\pi: \tilde{C} \rightarrow C$ of smooth connected nonhyperelliptic curves, with $g(C)=3$, and $g(\tilde{C})=5$, and let $P=P(\tilde{C} / C)$ denote the two dimensional Prym variety of $\tilde{C} / C$. Up to choice of origin, this p.p.a.v. (principally polarized abelian variety) can be constructed as follows (Mumford [Mu1, p. 342]): The double cover $\pi: \tilde{C} \rightarrow C$ determines a norm map $\mathrm{Nm}: \operatorname{Pic}^{d}(\tilde{C}) \rightarrow \operatorname{Pic}^{d}(C)$ for every $d$. For $d=2 g(C)-2=4$, we consider $\mathrm{Nm}: \operatorname{Pic}^{4}(\tilde{C}) \rightarrow \operatorname{Pic}^{4}(C)$ and the inverse image $\mathrm{Nm}^{-1}\left(K_{C}\right)$ of the canonical line bundle $K_{C}$ of $C$. This inverse image has exactly two connected components, $\mathrm{Nm}^{-1}\left(K_{C}\right)=P^{+} \cup P^{-}$, according to the parity of the number of sections of the corresponding line bundles. In particular, we take $P=P^{+}=$\{line bundles $L$ on $\tilde{C}$ with an even number of sections, and such that $\operatorname{Nm}(L)=K_{C}$, to be the Prym variety $P(\tilde{C} / C)$ (except for a choice of origin). The theta divisor on $P$ is given by the underlying reduced variety $\Xi$ of the intersection divisor $P^{+} \cap \tilde{\Theta}=2 \Xi$, where $\tilde{\Theta}=\{$ effective line bundles of degree 4 on $\tilde{C}\}, \Xi=\left\{\right.$ line bundles $L$ on $\tilde{C}$, with $h^{0}(L)>0$ and even, such that $\left.\operatorname{Nm}(L)=K_{C}\right\}$. Then $(P, \Xi)$ is a two dimensional p.p.a.v., $\Xi$ is isomorphic to a smooth genus two curve $\Sigma$, and $(P, \Xi)$ is isomorphic to the Jacobian variety $(J(\Sigma), \Sigma)$ of $\Sigma$, except for lack of a choice of origin. The restriction of the canonical involution $\mu \mapsto\left(K_{\tilde{C}}-\mu\right)$ from $\operatorname{Pic}^{4}(\tilde{C})$ to $P \cong \operatorname{Pic}^{1}(\Sigma)$ preserves $\Sigma$ and corresponds to the involution $\mu \mapsto\left(K_{\Sigma}-\mu\right)$ of $\operatorname{Pic}^{1}(\Sigma)$, and to the involution -id of $(J(\Sigma), \Sigma)$. (The natural involution of $\mathrm{Pic}^{2 g-2}$ taking $D$ to $\tilde{K}-D$ always restricts on $P^{+}$to a translate of the natural involution -id, hence on $\Sigma$ to the canonical involution.)

\section{The Abel parameterization $\varphi: X \rightarrow \Xi$ of a Prym theta divisor, and the restricted norm map $h: X \rightarrow\left|K_{C}\right| \stackrel{\sim}{=} \mathbb{P}^{2 *}$.}

Now recall the construction of the variety $X$ parametrizing the Prym theta divisor $\Xi$. Let $C$ be a smooth connected nonhyperelliptic curve of genus three and $\tilde{C} \rightarrow C$ an étale connected double cover, where $\tilde{C}$ has genus five, and $\Xi \subset P \subset \operatorname{Pic}^{4}(\tilde{C})$ the embedded Prym variety constructed above. The Abel-Jacobi map $\varphi: \tilde{C}^{(4)} \rightarrow \tilde{\Theta}$ is a resolution of singularities of $\tilde{\Theta}$, a local 
isomorphism over smooth points of $\tilde{\Theta}$, and with a $\mathbb{P}^{1}$ fiber over each point of the curve $\operatorname{sing}(\tilde{\Theta})$. Since $h^{0}(L) \geq 2$ for $L$ in $\Xi$, by Riemann's singularity theorem $\Xi \subset \operatorname{sing}(\tilde{\Theta})$, and if we define $X=\varphi^{-1}(\Xi) \subset \tilde{C}^{(4)}$, then $\varphi: X \rightarrow \Xi$ is a $\mathbb{P}^{1}$ bundle over the genus two curve $\Xi=\Sigma$.

The other map of interest to us is the restriction $h$ to $X$, of the finite norm map Nm : $\tilde{C}^{(4)} \rightarrow C^{(4)}$. The map $h: X \rightarrow\left|K_{C}\right| \cong \mathbb{P}^{2 *}$, takes a point $D=p_{1}+p_{2}+p_{3}+p_{4}$ of $X$, to the canonical divisor $\bar{D}=\bar{p}_{1}+\bar{p}_{2}+\bar{p}_{3}+\bar{p}_{4}$ on $C$, where $\pi\left(p_{i}\right)=\bar{p}_{i}$. The norm map Nm : $\tilde{C}^{(4)} \rightarrow C^{(4)}$ has degree $2^{4}=16$, but is branched over any divisor $\bar{D}$ having multiplicities, i.e. in which some $\bar{p}_{i}$ equals some $\bar{p}_{j}$. We claim that $h$ has degree 8 and is branched precisely over the dual curve $C^{*} \subset\left|K_{C}\right|$. By Mumford's theory in [Mu1, Mu2, W], exactly half the divisors of $\tilde{C}^{(4)}$ over a given point of $C^{(4)}$ have even parity, so $h$ has degree 8 . Since all effective canonical divisors of $C$ are cut out on the canonical plane embedding of $C$ in $\left|K_{C}\right|^{*}$ by lines, $h$ is branched at most over $C^{*}=$ lines cutting divisors on $C$ with multiplicities. Consider a general such canonical divisor with multiplicities $\bar{D}=2 \bar{p}+\bar{r}+\bar{s}$ on $C$. The ramified divisors over it in $\tilde{C}^{(4)}$ have form $p+p^{\prime}+r+s$, where $p, p^{\prime}$ are the preimages of $p$, and $r, s$ are preimages of $\bar{r}, \bar{s}$, under the double cover $\pi: \tilde{C} \rightarrow C$. If $r^{\prime}$, $s^{\prime}$ are the other preimages of $\bar{r}, \bar{s}$, then by Mumford's theory, $p+p^{\prime}+r+s$, and $p+p^{\prime}+r+s^{\prime}$, have opposite parity, as do $p+p^{\prime}+r+s$, and $p+p^{\prime}+r^{\prime}+s$. Thus there are exactly two ramification divisors over $\bar{D}$ with even parity, which we may assume are $p+p^{\prime}+r+s$, and $p+p^{\prime}+r^{\prime}+s^{\prime}$. Hence over a smooth point on $C^{*}$ in $\left|K_{C}\right|$, corresponding to the canonical divisor $\bar{D}=2 \bar{p}+\bar{r}+\bar{s}$ on $C$, there are exactly the two simple ramification points $D=p+p^{\prime}+r+s$, and $D^{\prime}=p+p^{\prime}+r^{\prime}+s^{\prime}$, of $h: X \rightarrow\left|K_{C}\right|$.

Since the map $h: X \rightarrow\left|K_{C}\right|$ recovers the dual curve $C^{*}$ as its branch locus, it recovers also $C$ as the dual of the branch locus, when $C$ is nonhyperelliptic. To recover $\tilde{C} / C$ from $X$ we will show how to recover the map $h$ from $X$, and then also the double cover $\pi: \tilde{C} \rightarrow C$ from $h$. Since $\Sigma$ has genus two, any map of $\mathbb{P}^{1}$ to $\Sigma$ is constant, so $X$ contains a unique family of copies of $\mathbb{P}^{1}$ hence has only one structure of $\mathbb{P}^{1}$ bundle. Thus we can already recover $\Xi$ and the $\mathbb{P}^{1}$ bundle map $\varphi: X \rightarrow \Xi$, from $X$. In fact, as Yin shows in $[\mathbf{Y}], \varphi: X \rightarrow \Xi \subset P$ is equivalent to an Albanese map for $X$.

In passing, we calculate the genus of the inverse image curve over a general line $H$ in $\left|K_{C}\right|$, by the map $h$. Since h has degree 8 , the branch locus $C^{*}$ has degree 12, and there are two ramification points of $h$ over each general branch point, by Riemann-Hurwitz the genus of the preimage $h^{-1}(H)$ is $(1 / 2)[2+8(-2)+24]=5$. 


\section{The line bundle $\mathcal{O}_{X}(1)=h^{*}(\mathcal{O}(1))$.}

Next we recover the line bundle giving rise to the restricted norm map $h$, in terms of the Abel map $\varphi$, as follows: Let $\mathcal{O}_{X}(1)=h^{*}(\mathcal{O}(1))$, be the pullback to $X$, of the line bundle $\mathcal{O}_{\left|K_{C}\right|}(1)$ on the canonical space $\left|K_{C}\right|$. We claim:

Proposition 1. $\mathcal{O}_{X}(1)=\mathcal{T}_{\varphi} \otimes \varphi^{*}\left(K_{\Xi}\right)$, where $\mathcal{T}_{\varphi}$ is the relative tangent sheaf of $\varphi(=$ the sheaf of tangents along the fibers of $\varphi)$, and $\varphi^{*}\left(K_{\Xi}\right)$ is the pullback of the canonical sheaf on the genus two curve $\Xi$.

Proof. Consider the line bundle $\mathcal{O}_{X}(1)$ restricted to one fiber of the $\mathbb{P}^{1}$ bundle $\varphi: X \rightarrow \Xi$. We will show this restriction has degree two by intersecting a divisor of $\mathcal{O}_{X}(1)$ with a fiber of $\varphi$. Consider first a divisor of $\mathcal{O}_{\left|K_{C}\right|}(1)$ of form $H_{\bar{p}}$, consisting of all canonical divisors on $C$ containing a given point $\bar{p}$. This is represented by a line $H_{\bar{p}}$, tangent to $C^{*}$. The pullback of this divisor to $X$ has two components, $h^{*}\left(H_{\bar{p}}\right)=D_{p}+D_{p^{\prime}}$, represented by divisors on $\tilde{C}$ which contain respectively either $p$ or $p^{\prime}$, the two preimages of $\bar{p}$ in $\tilde{C}$ under $\pi: \tilde{C} \rightarrow C$. Since $C^{*}$ is irreducible of degree 12 when $C$ is smooth, $H_{\bar{p}}$ is not contained in the branch locus $C^{*}$ and $h$ is unramified at a general point of each component of $h^{*}\left(H_{\bar{p}}\right)=D_{p}+D_{p^{\prime}}$, so this fiber is reduced. Now each fiber of $\varphi: X \rightarrow \Xi$ is a pencil of divisors of degree 4 moving in a linear series on $\tilde{C}$, without fixed points, $(\tilde{C}$ is not trigonal since the singular locus of $\tilde{\Theta}$ contains the curve $\Xi$ of genus two, whereas for $\tilde{C}$ trigonal the singular locus of $\tilde{\Theta}$ is two copies of $\tilde{C}$ by [A-M, p. 212]). Hence exactly one divisor in each pencil contains $p$, and exactly one contains $p^{\prime}$. Thus in general $h^{*}\left(H_{\bar{p}}\right)$ meets each fiber of $\varphi$ twice, and the degree of the restriction of $\mathcal{O}_{X}(1)$ to each fiber is 2 .

Now the restriction of $\mathcal{T}_{\varphi}$ to a fiber of $\varphi$, is the tangent sheaf of a projective line, hence also of degree 2 . Thus the tensor product $\mathcal{O}_{X}(1) \otimes \mathcal{T}_{\varphi}^{*}$ has degree zero on the fibers, and thus is the pullback $\varphi^{*}(\mathcal{L})$ of a sheaf $\mathcal{L}$ from $\Xi$ by the map $\varphi: X \rightarrow \Xi$. Since for each point $p$ on $\tilde{C}, D_{p}$ has been seen to be generically a section of $\varphi$, by Zariski's Main Theorem $\varphi$ restricts on $D_{p}$ to an isomorphism of $D_{p}$ with $\Xi$, so we can compute $\mathcal{L}$ by restricting $\mathcal{O}_{X}(1) \otimes \mathcal{T}_{\varphi}^{*}$ to the section $D_{p}$. The tangent sheaf $\mathcal{T}_{\varphi}$ to the fibers of $\varphi$ is the normal sheaf to a section $D_{p}$, so $\mathcal{T}_{\varphi}^{*}$ restricts on $D_{p}$ to $\left.\mathcal{O}\left(-D_{p}\right)\right|_{D_{p}}$, while $\mathcal{O}_{X}(1)=\mathcal{O}\left(D_{p}+D_{p^{\prime}}\right)$. Thus $\mathcal{O}_{X}(1) \otimes \mathcal{T}_{\varphi}^{*}$ restricts on $D_{p}$ to $\left.\mathcal{O}\left(D_{p^{\prime}}\right)\right|_{D_{p}}$. To compute this, we must intersect $D_{p^{\prime}}$ with $D_{p}$. This intersection is made up of points of $X$ representing divisors (on $\tilde{C}$ ) which contain both $p$ and $p^{\prime}$. Such a divisor must lie over a divisor on $C$ of form $2 \bar{p}+\bar{r}+\bar{s}$. Hence the divisor on $\tilde{C}$ is a ramification divisor of $h$ of form $p+p^{\prime}+r+s$, or $p+p^{\prime}+r^{\prime}+s^{\prime}$, as we have computed before in Section 2. Thus the intersection divisor $D_{p} \cdot D_{p^{\prime}}$ contains at most two points, and contains exactly two unless $r+s=r^{\prime}+s^{\prime}$, i.e. unless $s=r^{\prime}, s^{\prime}=r$. That would mean our ramification divisor $p+p^{\prime}+r+s=p+p^{\prime}+r+r^{\prime}$ lies over the divisor $2 \bar{p}+2 \bar{r}$, one 
of the 28 divisors cut by a bitangent line to the canonical plane quartic $C$. Thus for a general point $p$ on $\tilde{C}, D_{p} \cdot D_{p^{\prime}}$ contains exactly two points. To see that the two intersection points are simple, note that the fiber $h^{-1}(H)$ over a general line $H$ has arithmetic genus 5 by the computation at the end of Section 2, so the reduced curve $h^{-1}\left(H_{\bar{p}}\right)=D_{p} \cup D_{p^{\prime}}$ also has arithmetic genus 5, and is composed of two components, each isomorphic to the curve $\Xi$ of genus two. Since the components meet twice, both intersections are transverse. Thus the divisor $D_{p} \cdot D_{p^{\prime}}$ has degree 2 on $D_{p}$, and (as can be seen from the explicit description of its points above) is invariant for the involution $\iota$ on $X$ induced by the involution $\iota$ of $\tilde{C}$ associated to $\pi: \tilde{C} \rightarrow C$.

Since the full inverse image of a canonical divisor $\bar{p}+\bar{q}+\bar{r}+\bar{s}$ on $C$ under the unramified double cover $\pi$, is a canonical divisor $p+p^{\prime}+q+$ $q^{\prime}+r+r^{\prime}+s+s^{\prime}$ on $\tilde{C}$, the involution $\iota$ on $X$ takes $(p+q+r+s)$ to $p^{\prime}+q^{\prime}+r^{\prime}+s^{\prime}=K_{\tilde{C}}-(p+q+r+s)$. Thus $\iota$ corresponds to the canonical involution $\mu \mapsto\left(K_{\tilde{C}}-\mu\right)$ on $\operatorname{Pic}^{4}(\tilde{C})$, which on $\Xi$ must be the hyperelliptic one, i.e. on $\Xi=\{$ effective divisors of degree one on $\Xi\}$, the involution taking $\mu$ to $\left(K_{\Xi}-\mu\right)$.

Since the divisor cut by $D_{p^{\prime}}$ on $D_{p}$ is invariant under the involution of $X$, it corresponds under the isomorphism $\varphi: D_{p} \rightarrow \Xi$, to an invariant divisor for the canonical hyperelliptic involution on $\Xi$, hence to a canonical divisor on $\Xi$. Thus $\mathcal{O}_{X}(1) \otimes \mathcal{T}_{\varphi}^{*}$ restricts on $D_{p}$ to $\varphi^{*}\left(K_{\Xi}\right)$, hence $\mathcal{O}_{X}(1) \otimes \mathcal{T}_{\varphi}^{*} \cong \varphi^{*}\left(K_{\Xi}\right)$ also on $X$, and thus $\mathcal{O}_{X}(1)=\mathcal{T}_{\varphi} \otimes \varphi^{*}\left(K_{\Xi}\right)$.

Corollary 1. Since $X$ determines the unique $\mathbb{P}^{1}$ bundle structure $\varphi: X \rightarrow$ $\Xi$, it determines also $\mathcal{T}_{\varphi}, \varphi^{*}\left(K_{\Xi}\right)$, and hence $\mathcal{O}_{X}(1)=\mathcal{T}_{\varphi} \otimes \varphi^{*}\left(K_{\Xi}\right)$, the line bundle associated to the map $h: X \rightarrow\left|K_{C}\right|$.

\section{Completeness of the linear system defining $h$, and the recovery of $\pi: \tilde{C} \rightarrow C$ from $X$.}

We claim the map $h: X \rightarrow\left|K_{C}\right|$ is given by a complete linear system, and hence the map $h$ itself is determined by $X$. To see this, we push $\mathcal{O}_{X}(1)$ down to $\Xi$ by $\varphi$, to get $\varphi_{*}\left(\mathcal{O}_{X}(1)\right) \cong \varphi_{*}\left(\mathcal{T}_{\varphi} \otimes \varphi^{*}\left(K_{\Xi}\right)\right) \cong \varphi_{*}\left(\mathcal{T}_{\varphi}\right) \otimes K_{\Xi}$. Then using Serre duality, $h^{0}\left(\varphi_{*}\left(\mathcal{O}_{X}(1)\right)\right)=h^{0}\left(\varphi_{*}\left(\mathcal{T}_{\varphi}\right) \otimes K_{\Xi}\right)=h^{1}\left(\varphi_{*}\left(\mathcal{T}_{\varphi}\right)^{*}\right)$. In [Y], H. Yin shows that $\varphi_{*}\left(\mathcal{T}_{\varphi}\right)$ is canonically isomorphic to the self dual bundle $\operatorname{End}_{0}(\triangle)$ of trace zero endomorphisms of $\triangle$, where $\triangle$ is the direct image of a Poincaré line bundle as described below, and computes $h^{1}\left(\varphi_{*}\left(\mathcal{T}_{\varphi}\right)\right)=3$. Hence $h^{0}\left(\mathcal{O}_{X}(1)\right)=h^{0}\left(\varphi_{*}\left(\mathcal{O}_{X}(1)\right)\right)=h^{0}\left(\varphi_{*}\left(\mathcal{T}_{\varphi}\right) \otimes K_{\Xi}\right)=h^{1}\left(\varphi_{*}\left(\mathcal{T}_{\varphi}\right)^{*}\right)=$ $h^{1}\left(\varphi_{*}\left(\mathcal{T}_{\varphi}\right)\right)=3$. Thus $X$ determines both the line bundle and the linear system defining the map $h: X \rightarrow\left|K_{C}\right|$, hence also $h$, its branch locus $C^{*}$ and thus also the curve $C$. (Later we will show the bundle $\triangle$ is stable and can be taken to have determinant $\mathcal{O}$, so then $\triangle$ is "simple", i.e. $h^{0}\left(\operatorname{End}_{0}(\triangle)\right)=0$, and Riemann Roch gives $\chi\left(\operatorname{End}_{0}(\triangle)\right)=\operatorname{degree}\left(\operatorname{End}_{0}(\triangle)\right)+\operatorname{rank}\left(\operatorname{End}_{0}(\triangle)\right)$. $(1-g)=0+3(1-2)=-3$. Hence $h^{1}\left(\operatorname{End}_{0}(\triangle)\right)=3+h^{0}\left(\operatorname{End}_{0}(\triangle)\right)=$ 
3, giving another computation of $h^{1}\left(\varphi_{*}\left(\mathcal{T}_{\varphi}\right)\right)$, modulo the isomorphism of $\varphi_{*}\left(\mathcal{T}_{\varphi}\right)$ with $\operatorname{End}_{0}(\triangle)$.)

To get the double cover $\tilde{C} \rightarrow C$, look again at the components of the inverse images of the lines $H_{\bar{p}}$ tangent to the branch locus $C^{*}$. These components are parametrized by the points of $\tilde{C}$. I.e. the map taking $p$ on $\tilde{C}$ to the component $D_{p}$ of divisors in $h^{-1}\left(H_{\bar{p}}\right)$ containing $p$, is birational from $\tilde{C}$ to the curve of components of inverse images of tangent lines $H_{\bar{p}}$ to $C^{*}$, and this birational map takes the involution on $\tilde{C}$ to that on $X$. Thus this curve of components determines both the curve $\tilde{C}$, and the involution defining the double cover $\pi: \tilde{C} \rightarrow C$. Thus $X$ determines $\pi: \tilde{C} \rightarrow C$.

Note. Since we showed in $\S 3$ that $D_{p}$ is a section of $\varphi: X \rightarrow \Sigma$, the map taking $p$ to $D_{p}$ represents $\tilde{C}$ as a curve in the variety of sections of $\varphi$ with self intersection equal to $D_{p} \cdot D_{p}=\operatorname{deg}\left(\left.\mathcal{T}_{\varphi}\right|_{D_{p}}\right)=\operatorname{deg}\left(\mathcal{O}_{X}(1) \otimes\right.$ $\left.\varphi^{*}\left(K_{\Sigma}^{*}\right)\right)\left.\right|_{D_{p}}=\operatorname{deg}\left(\left.h\right|_{D_{p}}\right)+\operatorname{deg}\left(K_{\Sigma}^{*}\right)=4-2=2$. Yin shows [Y, p. 23] that this representation of $\tilde{C}$ is birational onto a component $Z$ of the Hilbert scheme $S_{2}$ of all sections of $X$ of self intersection 2. From the next section it will follow that $Z$ is the unique component of $S_{2}$.

\section{Relationship between the correspondence $\tilde{C} / C \mapsto X$ and that of Narasimhan-Ramanan and Verra.}

Now consider the moduli space $\mathcal{M}$ of $S$-equivalence classes of semi-stable rank 2 vector bundles of determinant $\mathcal{O}$, over the smooth curve $\Sigma$ of genus 2, and the isomorphism $\mathcal{M} \cong|2 \Theta|$, where $\Theta=\Sigma \subset \operatorname{Pic}^{1}(\Sigma)$. Recall that to the bundle $\mathcal{E}$ Narasimhan-Ramanan associate the subset $D_{\mathcal{E}}=\left\{\xi\right.$ in $\operatorname{Pic}^{1}(\Sigma)$ : $\left.H^{0}(\mathcal{E} \otimes \xi) \neq 0\right\} \subset \operatorname{Pic}^{1}(\Sigma)$, the support of a unique divisor $\tilde{C}_{\mathcal{E}}$ linearly equivalent to $2 \Theta$, and that a nonstable bundle $S$-equivalent to $\mu \oplus \mu^{-1}$ with $\mu$ in $\operatorname{Pic}^{0}(\Sigma)$ corresponds to the point $\Theta_{\mu}+\Theta_{\mu^{-1}}$ on the Kummer surface $\mathcal{K}$ in $|2 \Theta|$. Then Verra's map $\tilde{C}_{\mathcal{E}} \mapsto\left(\tilde{C}_{\mathcal{E}}, \iota\right)$ carries the complement of $\mathcal{K}$ in $|2 \Theta|$ onto the set of irreducible stable double covers $(\tilde{C}, \iota)$ in $\mathcal{P}^{-1}(J(\Sigma))$ such that $\tilde{C}$ is not hyperelliptic. Composing gives a rational map $\mathcal{M} \rightarrow-$ $\mathcal{P}^{-1}(J(\Sigma))$, taking stable bundles to irreducible stable double covers, which factors through the quotient of $\mathcal{M}$ by the group $G$ of points of order 2 in $\operatorname{Pic}^{0}(\Sigma)$, i.e. by a rational map taking stable $\mathbb{P}^{1}$ bundles to irreducible stable double covers. We will show that the Abel parametrization studied by Yin gives a birational inverse to this map. I.e. let $\tilde{C} / C$ be a double cover of a smooth, nonhyperelliptic, genus three curve, and $\varphi: X \rightarrow \Xi$ the associated Abel parametrization of the theta divisor on the Prym variety $(P, \Xi) \cong(J(\Sigma), \Sigma)=$ the Jacobian of a smooth genus two curve $\Sigma$. If $\mathcal{E}$ is a rank 2 vector bundle of determinant $\mathcal{O}$ on $\Sigma$ with $\mathbb{P}(\mathcal{E}) \cong X$, then $\mathcal{E}$ is stable and the associated divisor $\tilde{C}_{\mathcal{E}}$ in $|2 \Xi|$ is isomorphic to $\tilde{C}$ (preserving the involution). Conversely, if $\mathcal{E}$ is a stable bundle on $\Sigma$ with associated divisor 
$\tilde{C}$ in $\operatorname{Pic}^{1}(\Sigma)$, where $\tilde{C}$ is smooth and nonhyperelliptic, then $\mathbb{P}(\mathcal{E}) \cong X$, the Abel parametrization associated to the Prym theta divisor $=$ of $(\tilde{C}, \iota)$.

First we recall some basic facts about bundles:

1) Definition: For a rank 2 vector bundle $\mathcal{E}, \operatorname{deg}(\mathcal{E})=\operatorname{deg}(\operatorname{det}(\mathcal{E}))$. $\mathcal{E}$ is "stable" (resp. semi-stable) if and only if for every sub-line bundle $\mathcal{L} \subset \mathcal{E}$, $\operatorname{deg}(\mathcal{L})<(1 / 2) \operatorname{deg}(\mathcal{E}),($ resp. $\operatorname{deg}(\mathcal{L}) \leq(1 / 2) \operatorname{deg}(\mathcal{E})) . \mathbb{P}(\mathcal{E})$ is stable if and only if $\mathcal{E}$ is stable.

2) If $\mathcal{E}, \tilde{\mathcal{E}}$ are rank 2 vector bundles on $\Sigma$, then $\mathbb{P}(\mathcal{E}) \cong \mathbb{P}(\tilde{\mathcal{E}})$ if and only if $\mathcal{E} \cong \tilde{\mathcal{E}} \otimes \mathcal{L}$ for some line bundle $\mathcal{L}$.

3) Every $\mathbb{P}^{1}$ bundle on $\Sigma$ has form $\mathbb{P}(\mathcal{E})$ for some rank 2 vector bundle $\mathcal{E}$.

4) Since $\operatorname{det}(\mathcal{E} \otimes \mathcal{L})=\operatorname{det}(\mathcal{E}) \otimes \mathcal{L}^{2}$, a $\mathbb{P}^{1}$ bundle $\varphi: X \rightarrow \Sigma$ has form $\mathbb{P}(\mathcal{E}) \cong X$ for some $\mathcal{E}$ with $\operatorname{det}(\mathcal{E}) \cong \mathcal{O}$ if and only if $X \cong \mathbb{P}(\tilde{\mathcal{E}})$ for some $\tilde{\mathcal{E}}$ with $\operatorname{deg}(\tilde{\mathcal{E}})$ even.

In Yin's treatment of the $\mathbb{P}^{1}$ bundle $\varphi: X \rightarrow \Xi \subset \operatorname{Pic}^{4}(\tilde{C})$, he considers a Poincaré bundle $\mathcal{L}$ on $\tilde{C} \times \operatorname{Pic}^{4}(\tilde{C})$, i.e. a line bundle $\mathcal{L}$ such that for each $\nu$ in $\operatorname{Pic}^{4}(\tilde{C})$, we have $\left.\mathcal{L}\right|_{\tilde{C} \times\{\nu\}} \cong \nu$. If we denote the restriction to $\tilde{C} \times \Sigma$ also by $\mathcal{L}$, and the second projection by $\mu$, he puts $\mathcal{E}=\mu_{*}(\mathcal{L})$ so that $\mathcal{E}$ is a rank two vector bundle on $\Sigma$ such that $X \cong \mathbb{P}(\mathcal{E})$.

Lemma 1. Given a stable rank 2 vector bundle $\mathcal{E}$ with $\operatorname{det}(\mathcal{E}) \cong \mathcal{O}$, on a smooth genus 2 curve $\Sigma$, and associated $\mathbb{P}^{1}$ bundle $X=\mathbb{P}(\mathcal{E})$, the natural map from $\left\{\right.$ sections $\sigma$ of $X \rightarrow \Sigma$, such that $\left.\left(\sigma^{2}\right)=2\right\}$ to $\left\{\xi\right.$ in $\operatorname{Pic}^{1}(\Sigma)$ : $\left.H^{0}(\mathcal{E} \otimes \xi) \neq 0\right\}$ is a bijection.

Proof. A section $\sigma: \Sigma \rightarrow X$ is equivalent to a sub-line bundle $\mathcal{L} \subset \mathcal{E}$, where for each point $p$ the fiber $\mathcal{L}_{p}$ defines $\sigma(p)$. The map from the set of sections to $\operatorname{Pic}(\Sigma)$ takes $\sigma$ to the abstract (dual) line bundle $\mathcal{L}^{*}$, forgetting the inclusion $\mathcal{L} \subset \mathcal{E}$. We show next that if $\left(\sigma^{2}\right)=2$, then $\operatorname{deg}\left(\mathcal{L}^{*}\right)=1$. Since $\left(\sigma^{2}\right)=\operatorname{deg}\left(N_{\sigma} / X\right)$, we want to calculate the degree of the normal bundle to $\sigma$ in $X$. Now $N_{\sigma} / X \cong \mathcal{H o m}(\mathcal{L}, \mathcal{E} / \mathcal{L}) \cong \mathcal{H} \operatorname{om}\left(\mathcal{L}, \mathcal{L}^{*}\right) \cong\left(\mathcal{L}^{*}\right)^{2}$, so $\left(\sigma^{2}\right)=2 \operatorname{deg}\left(\mathcal{L}^{*}\right)$, and $\left(\sigma^{2}\right)=2 \operatorname{implies} \operatorname{deg}\left(\mathcal{L}^{*}\right)=1$. (We have used $\mathcal{O} \cong \operatorname{det}(\mathcal{E}) \cong \mathcal{L} \otimes(\mathcal{E} / \mathcal{L})$ to deduce $\mathcal{E} / \mathcal{L} \cong \mathcal{L}^{*}$.) Next, if $0 \rightarrow \mathcal{L} \rightarrow \mathcal{E}$ is exact, then tensoring with $\mathcal{L}^{*}$ gives $0 \rightarrow \mathcal{O} \rightarrow \mathcal{E} \otimes \mathcal{L}^{*}$ exact, so the image of 1 defines a nonzero section of the sheaf $\mathcal{E} \otimes \mathcal{L}^{*}$. Thus the forgetful map from $\left\{\right.$ sections $\sigma$ of $X \rightarrow \Sigma$, such that $\left.\left(\sigma^{2}\right)=2\right\}$ to $\left\{\xi\right.$ in $\left.\operatorname{Pic}^{1}(\Sigma): H^{0}(\mathcal{E} \otimes \xi) \neq 0\right\}$ is well defined.

To prove surjectivity, if $\xi$ is in $\operatorname{Pic}^{1}(\Sigma)$, and $H^{0}(\mathcal{E} \otimes \xi) \neq 0$, there is a nonzero homomorphism $\mathcal{O} \rightarrow \mathcal{E} \otimes \xi$ sending 1 to a nonzero global section $s$ of $\mathcal{E} \otimes \xi$, so multiplication by this section gives a nonzero homomorphism $\xi^{*} \rightarrow \mathcal{E}$. This is an injective sheaf map since $\mathcal{O}$ is a sheaf of integral domains, and defines an injection at least on those fibers of the line bundle $\xi^{*}$ at points where s does not vanish. This defines a rational section $\Sigma \rightarrow X$ of the $\mathbb{P}^{1}$ bundle $X=\mathbb{P}(\mathcal{E})$ which extends uniquely to a regular section $\sigma$, hence defines a unique sub-line bundle $\mathcal{L} \subset \mathcal{E}$ such that $\xi^{*} \subset \mathcal{L} \subset \mathcal{E}$ and $\mathcal{E} / \mathcal{L}$ is 
a line bundle. Since $\mathcal{E}$ is stable, $-1=\operatorname{deg}\left(\xi^{*}\right) \leq \operatorname{deg}(\mathcal{L})<\operatorname{deg}(\mathcal{E}) / 2=0$. Thus $\operatorname{deg}(\mathcal{L})=-1$, hence $\mathcal{L}=\xi^{*}$, and $\mathcal{L}^{*} \cong \xi$, proving surjectivity.

To prove injectivity, we must show for each line bundle $\xi$ in $\operatorname{Pic}^{1}(\Sigma)$ there is at most one sub-line bundle of $\mathcal{E}$ which is the image of an inclusion $\xi^{*} \rightarrow \mathcal{E}$. It suffices to show that the space of homomorphisms $\operatorname{Hom}\left(\xi^{*}, \mathcal{E}\right)$ is at most one dimensional. Adapting an argument of $[\mathbf{N}-\mathbf{R}]$, if $\operatorname{dim}\left(\operatorname{Hom}\left(\xi^{*}, \mathcal{E}\right)\right) \geq 2$, and if the evaluation map $\xi^{*} \otimes \operatorname{Hom}\left(\xi^{*}, \mathcal{E}\right) \rightarrow \mathcal{E}$, were injective on fibers, then we would have $\mathcal{E} \cong \xi^{*} \oplus \xi^{*}$, which is impossible since $\operatorname{det}(\mathcal{E}) \cong \mathcal{O}$. Thus for some $p$ in $\Sigma$, there would be a nonzero sheaf homomorphism $\xi^{*} \rightarrow \mathcal{E}$ which is zero on the fiber $\xi_{p}^{*}$. But we have just argued in proving surjectivity that when $\mathcal{E}$ is stable, and $\operatorname{deg}(\xi)=1$, any nonzero map $\xi^{*} \rightarrow \mathcal{E}$ has a line bundle quotient, hence is injective on all fibers.

Corollary 2. If $C$ is nonhyperelliptic, the $\mathbb{P}^{1}$ bundle $X \rightarrow \Xi$ arising from the Abel parametrization of the Prym theta divisor $\Xi(\tilde{C} / C)$ is stable.

Proof. We showed in the note at the end of $\S 4$ that $X$ has sections $\sigma$ with $\left(\sigma^{2}\right)=2$, and we want to deduce that if $X=\mathbb{P}(\mathcal{E})$, then $\operatorname{deg}(\mathcal{E})$ is even. If $\mathcal{L} \subset \mathcal{E}$ is a sub-line bundle corresponding to a section $\sigma$ of $\mathcal{E}$, yielding an exact sequence $0 \rightarrow \mathcal{L} \rightarrow \mathcal{E} \rightarrow \mathcal{N} \rightarrow 0$, then $\operatorname{det}(\mathcal{E}) \cong \mathcal{L} \otimes \mathcal{N}$, and as above $\left(\sigma^{2}\right)=\operatorname{deg}(\operatorname{Hom}(\mathcal{L}, \mathcal{N}))=\operatorname{deg}\left(\mathcal{L}^{*} \otimes \mathcal{N}\right)=\operatorname{deg}(\mathcal{N})-\operatorname{deg}(\mathcal{L})$. Hence $\operatorname{deg}(\mathcal{E})=\operatorname{deg}(\mathcal{L} \otimes \mathcal{N})=\operatorname{deg}(\mathcal{L})+\operatorname{deg}(\mathcal{N})=\left(\sigma^{2}\right)+2 \operatorname{deg}(\mathcal{L}) \equiv\left(\sigma^{2}\right) \bmod 2$, so $\left(\sigma^{2}\right)=2$ implies $\operatorname{deg}(\mathcal{E})$ is even. Now the line bundle on $\tilde{C} \times \Sigma$ used in Yin's construction of $\mathcal{E}$ is only defined up to tensoring with the pullback of a line bundle $\mathcal{N}$ from $\Sigma$, so by the projection formula, $\mathcal{E}$ may be replaced by $\mathcal{E} \otimes \mathcal{N}$ where $\mathcal{N}^{-2}=\operatorname{det}(\mathcal{E})$, so we may assume $\operatorname{det}(\mathcal{E}) \cong \mathcal{O}$.

Next we show $\mathcal{E}$ is semi-stable. Any section $\sigma$ of $\mathbb{P}(\mathcal{E}) \cong X$ of self intersection 2 defines a sub-line bundle $\xi^{-1} \subset \mathcal{E}$ and an exact sequence $0 \rightarrow \xi^{-1} \rightarrow \mathcal{E} \rightarrow \xi \rightarrow 0$, with $2=\left(\sigma^{2}\right)=2 \operatorname{deg}(\xi)$, so that $\xi$ belongs to $\operatorname{Pic}^{1}(\Sigma)$. If $\mathcal{E}$ is not semi-stable, and $\mathcal{L}$ is a line bundle of positive degree mapping into $\mathcal{E}$, it cannot map into $\xi^{-1}$ hence maps nontrivially into $\xi$ by composition, hence has degree exactly 1 . Thus $\mathcal{L} \cong \xi$, and the sequence splits, i.e. $\mathcal{E} \cong \xi^{-1} \oplus \xi$. We will show this is impossible by showing it contradicts the representation in $\S 4$ of $\tilde{C}$ as a complete curve of sections of $X$ of self intersection 2. I.e. if $\mathcal{E} \cong \xi^{-1} \oplus \xi$, then we can represent $X \cong \mathbb{P}\left(\xi^{-1} \oplus \xi\right) \cong \mathbb{P}\left(\mathcal{O} \oplus \xi^{2}\right)$, so that $X$ is the completion of the line bundle $\xi^{2}$ by adding a section $S_{\infty}$ at infinity, and the section of $X$ corresponding to $\xi^{-1}$ corresponds to the zero section $S_{0}$ of the line bundle $\xi^{2}$.

Claim. If $X \cong \mathbb{P}\left(\mathcal{O} \oplus \xi^{2}\right)$, then all sections of $X$ of self intersection 2 correspond to elements of the vector space of regular sections of the line bundle $\xi^{2}$.

Proof. Regular sections of the line bundle give regular sections of the completion of the line bundle, i.e. of $X$. Conversely, we must show a section of 
$X$ misses the section $S_{\infty}$ at infinity. A section of $X$ is irreducible (since isomorphic to $\Sigma$ ), hence cannot contain the infinite section without equalling it. Since $S_{\infty}$ has self intersection -2, it cannot equal a regular section of self intersection 2, so must meet it properly. Since $S_{0}$ and $F$ (the zero section and a fiber), form a $\mathbb{Z}$ homology basis of $X$, a section $\sigma$ of $X$ is homologous to $a S_{0}+b F$, where $F \cdot S_{0}=1$, and $a, b$ are integers. Since $F \cdot \sigma=1$, and $F \cdot F=0$, we have $a=1$, so $\sigma=S_{0}+b F$, and $\left(\sigma^{2}\right)=2=2+2 b$. Thus $b=0, \sigma=S_{0}$ in homology, hence $\sigma \cdot S_{\infty}=0$. But since $\sigma$ meets the infinite section properly, $\sigma$ must be disjoint from $S_{\infty}$.

Since $\tilde{C}$ is complete, $\tilde{C}$ cannot map injectively into the affine space of all sections of $\mathbb{P}\left(\mathcal{O} \oplus \xi^{2}\right)$ of self intersection 2. Since by the argument in $\S 4, \tilde{C}$ does inject into the variety of sections of $X$ of self intersection 2, thus $\mathcal{E}$ is not isomorphic to $\xi^{-1} \oplus \xi$, and hence $\mathcal{E}$ is semi-stable. Now that we know $\mathcal{E}$ is semi-stable, the Narasimhan-Ramanan curve $D_{\mathcal{E}}=\{\xi$ in $\left.\operatorname{Pic}^{1}(\Sigma): H^{0}(\mathcal{E} \otimes \xi) \neq 0\right\}$, is defined and equals the support of a divisor $\tilde{C}_{\mathcal{E}}$ in $|2 \Theta|$. By Verra's explicit description of curves in $|2 \Theta|, D_{\mathcal{E}}$ is either reduced and irreducible of arithmetic genus five, or a union of one or two copies of $\Sigma$. Moreover we have maps $\tilde{C} \rightarrow Z \rightarrow D_{\mathcal{E}}$, where $Z$ is a curve of sections of $\varphi: X \rightarrow \Sigma$ of self intersection 2 , and $\tilde{C} \rightarrow Z$ is bijective. By [Y, Lemma 4, p. 23], the composition $\tilde{C} \rightarrow D_{\mathcal{E}}$ is a nonconstant finite map from $\tilde{C}$ to $D_{\mathcal{E}}$. We argue next this map is generically injective. By [Y, Lemma 3, p. 23], $Z$ is an irreducible component of the variety of sections of $X$ of self intersection 2. If $p \neq q$ are distinct points of $\tilde{C}$ corresponding to distinct sections $\sigma_{p} \neq \sigma_{q}$ of $X$, but the same line bundle $\xi_{p}=\xi_{q}$ in $\operatorname{Pic}^{1}(\Sigma)$, then $\xi_{p}^{-1}$ embeds onto more than one sub-line bundle of $\mathcal{E}$, so the vector space $\operatorname{Hom}\left(\xi_{p}^{-1}, \mathcal{E}\right)$ has dimension $\geq 2$. Thus by varying the embedding of $\xi_{p}$ in $\mathcal{E}$, there is a $\mathbb{P}^{1}$ of sections of $\mathrm{X}$ passing through the point $\sigma_{p}$ on $Z$, and all having self intersection 2 .

Since the component $Z$ is birational to $\tilde{C}$ of genus 5 , this can only occur for a finite number of points $p$, so the map $\tilde{C} \rightarrow D_{\mathcal{E}}$ is generically injective as claimed. Then the divisor $\tilde{C}$ of $|2 \Theta|$ supported on $D_{\mathcal{E}}$ is not one of the reducible divisors $\Theta_{\mu}+\Theta_{\mu^{-1}}$ on the Kummer surface, for $\mu$ in $\operatorname{Pic}^{0}(\Xi)$, which parametrize semi-stable, nonstable bundles of the form $\mu \oplus \mu^{-1}$. Hence $\mathcal{E}$ is in fact stable, and $D_{\mathcal{E}}$ is reduced irreducible of arithmetic genus five, and thus $\tilde{C} \rightarrow D_{\mathcal{E}}$ is an isomorphism.

It now follows from Lemma 1 that $Z$ is the only component of sections of $X$ of self intersection 2 , and both maps $\tilde{C} \rightarrow Z \rightarrow D_{\mathcal{E}}$ are isomorphisms. Consequently, the divisor $\tilde{C}_{\mathcal{E}}$ associated by $[\mathbf{N}-\mathbf{R}]$ to the $\mathbb{P}^{1}$ bundle $X=\mathbb{P}(\mathcal{E})$ is isomorphic to the original smooth genus five curve $\tilde{C}$, hence we have proved

Corollary 3. Yin's correspondence $\tilde{C} / C \mapsto X$ is right inverse to Narasimhan-Ramanan's correspondence $X=\mathbb{P}(\mathcal{E}) \mapsto \tilde{C}_{\mathcal{E}}$, (modulo the action of 
the group $G \cong\left(\mathbb{Z}_{2}\right)^{4}$ of points of order two in $\left.\operatorname{Pic}^{4}(\tilde{C})\right)$. (There are 16 choices of the vector bundle $\mathcal{E}$ on $\Sigma$ defining the same $\mathbb{P}^{1}$ bundle $X$, and 16 divisors $\tilde{C}_{\mathcal{E}}$ in $|2 \Theta|$ defining isomorphic curves, since $G$ acts on $|2 \Theta|$.)

Since the map $\tilde{C} / C \mapsto X$ is right inverse to N-R's, and theirs is known to be an isomorphism between $\mathcal{M}$ and $|2 \Theta|$, it follows that Yin's construction is also left inverse to N-R's, at least on an open subset of the set of stable bundles in $\mathcal{M}$. I.e. a stable bundle $\mathcal{E}$ yields an irreducible curve $\tilde{C}$, and if $\tilde{C}$ is smooth and nonhyperelliptic, and if $X$ is the $\mathbb{P}^{1}$ bundle over $\Xi$ defined in the Prym construction associated to $(\tilde{C}, \iota)$, then $X \cong \mathbb{P}(\mathcal{E})$.

Without assuming the result that the map of $[\mathbf{N}-\mathbf{R}]$ is an isomorphism we can partially recover it as follows: Given a stable vector bundle $\mathcal{E}$ of determinant $\mathcal{O}$ on a smooth genus two curve $\Sigma$, such that the divisor $\tilde{C}_{\mathcal{E}}$ in $|2 \Theta|$ defined by $[\mathbf{N}-\mathbf{R}]$ is smooth, it follows from $[\mathbf{V}]$ that the canonical involution $\iota$ of $\operatorname{Pic}^{1}(\Sigma)$ is fix point free on $\tilde{C}_{\mathcal{E}}$, and that $\tilde{C}_{\mathcal{E}}$ and $\tilde{C}_{\mathcal{E}} / \iota$ are both nonhyperelliptic. If we put $X=\mathbb{P}(\mathcal{E})$ and denote the $\mathbb{P}^{1}$ bundle map by $\varphi: X \rightarrow \Sigma$, then it follows from Lemma 1 above that the curve $Z$ of sections of $\varphi: X \rightarrow \Sigma$ of self intersection 2 is isomorphic to $\tilde{C}_{\mathcal{E}}$. Hence we may write $\tilde{C}_{\mathcal{E}}$ for $Z$ and consider the tautological map $\gamma: \tilde{C}_{\mathcal{E}} \times \Sigma \rightarrow X$ given by evaluating a section at a point in $\Sigma$, i.e. $\gamma(\sigma, y)=\sigma(y)$. Thus the points $y$ of $\Sigma$ parametrize a family of maps $\gamma_{y}: \tilde{C}_{\mathcal{E}} \rightarrow \varphi^{-1}(y) \cong \mathbb{P}^{1}$. Since these maps are limits of such maps in cases where we know $X$ arises from the Abel parametrization of the Prym theta divisor $\Xi \cong \Sigma$, and in those cases $\gamma_{y}$ is a degree 4 map $\tilde{C} \rightarrow \mathbb{P}^{1}$, it follows that here also degree $\left(\gamma_{y}\right)=4$. If $\mathcal{L}=\gamma^{*}\left(\mathcal{O}_{\mathcal{E}}(1)\right)$, the family of line bundles $\mathcal{L}_{y}$ on $\tilde{C}_{\mathcal{E}}$ for $y$ in $\Sigma$ thus defines a map $\Sigma \rightarrow \operatorname{Pic}^{4}\left(\tilde{C}_{\mathcal{E}}\right)$ with target in $W_{4}^{1}\left(\tilde{C}_{\mathcal{E}}\right)=\operatorname{sing}\left(\Theta\left(\tilde{C}_{\mathcal{E}}\right)\right)$. Since as above this map is a limit of embeddings of $\Sigma \cong \Xi=P^{+} \cap \tilde{\Theta} \subset \operatorname{Pic}^{4}(\tilde{C})$ of $\Sigma$ onto a Prym theta divisor, it follows that the limiting map is still nonconstant in our case. Since $\operatorname{sing}\left(\Theta\left(\tilde{C}_{\mathcal{E}}\right)\right)$ has only two components, one of genus 4 and one of genus 2 [ACGH, p. 274], it follows that the map $\Sigma \rightarrow \operatorname{Pic}^{4}\left(\tilde{C}_{\mathcal{E}}\right)$ is again an embedding onto the unique genus two component, i.e. onto the Prym theta divisor $\Xi$ associated to $\left(\tilde{C}_{\mathcal{E}}, \iota\right)$. Thus $\mathcal{L}=\gamma^{*}\left(\mathcal{O}_{\mathcal{E}}(1)\right)$ is the restriction to $\tilde{C}_{\mathcal{E}} \times \Sigma$ of a Poincaré line bundle for $\tilde{C}_{\mathcal{E}} \times \operatorname{Pic}^{4}\left(\tilde{C}_{\mathcal{E}}\right)$. It follows then that pushing down this $\mathcal{L}$ gives the $\mathbb{P}^{1}$ bundle $X\left(\tilde{C}_{\mathcal{E}}, \iota\right)$ coming from the Abel parametrization of $\Sigma \cong \Xi(\tilde{C})$, so to show $\mathbb{P}(\mathcal{E}) \cong X\left(\tilde{C}_{\mathcal{E}}, \iota\right)$ we must compare the push down of $\mathcal{L}$ with the original bundle $\mathcal{E}$.

Lemma 2. With notation as above, $\mu_{*}(\mathcal{L}) \cong \mu_{*}\left(\gamma^{*}\left(\mathcal{O}_{\mathcal{E}}(1)\right)\right) \cong \mathcal{E}$.

Proof. Look again at the maps $\gamma: \tilde{C}_{\mathcal{E}} \times \Sigma \rightarrow X=\mathbb{P}(\mathcal{E}), \varphi: X \rightarrow \Sigma$ and (their composition) the second projection $\varphi \circ \gamma=\mu: \tilde{C}_{\mathcal{E}} \times \Sigma \rightarrow \Sigma$. Then pull back the bundle $\mathcal{E}$ from $\Sigma$ to $\varphi^{*}(\mathcal{E})$ on $X$, and consider the fundamental exact sequence $0 \rightarrow \mathcal{O}_{\mathcal{E}}(-1) \rightarrow \varphi^{*}(\mathcal{E}) \rightarrow \mathcal{O}_{\mathcal{E}}(1) \rightarrow 0$, where the quotient sheaf is dual to $\mathcal{O}_{\mathcal{E}}(-1)$ because $\operatorname{det}(\mathcal{E}) \cong \mathcal{O}$. Then pull back this sequence 
by $\gamma$ to $\tilde{C} \times \Sigma$ getting $0 \rightarrow \gamma^{*}\left(\mathcal{O}_{\mathcal{E}}(-1)\right) \rightarrow \gamma^{*} \varphi^{*}(\mathcal{E}) \rightarrow \gamma^{*}\left(\mathcal{O}_{\mathcal{E}}(1)\right) \rightarrow 0$, and push that down to $\Sigma$ by $\mu$, getting a long exact sequence that begins with $0 \rightarrow \mu_{*}\left(\gamma^{*} \mathcal{O}_{\mathcal{E}}(-1)\right) \rightarrow \mu_{*}\left(\gamma^{*} \varphi^{*}(\mathcal{E})\right) \rightarrow \mu_{*} \gamma^{*}\left(\mathcal{O}_{\mathcal{E}}(1)\right)$. Since the line bundle $\gamma^{*}\left(\mathcal{O}_{\mathcal{E}}(-1)\right)$ restricts to have degree -4 on the fibers of $\mu$, the leftmost sheaf is zero and we have an injection of bundles $\mu_{*}\left(\gamma^{*} \varphi^{*}(\mathcal{E})\right) \rightarrow \mu_{*} \gamma^{*}\left(\mathcal{O}_{\mathcal{E}}(1)\right)$. Since $\gamma^{*}\left(\mathcal{O}_{\mathcal{E}}(1)\right)$ restricts to line bundles of degree 4 with two sections on the fibers of $\mu$, the second bundle is also a 2-plane bundle, and the map is an isomorphism. Since by the projection formula $\mu_{*}\left(\gamma^{*} \varphi^{*}(\mathcal{E})\right) \cong \mathcal{E}$, we have $\mathcal{E} \cong \mu_{*}\left(\gamma^{*} \varphi^{*}(\mathcal{E})\right) \cong \mu_{*} \gamma^{*}\left(\mathcal{O}_{\mathcal{E}}(1)\right)=\mu_{*}(\mathcal{L})$.

Remark. Since by hypothesis $\operatorname{det}(\mathcal{E}) \cong \mathcal{O}$, not only is $\mathbb{P}(\mathcal{E}) \cong X\left(\tilde{C}_{\mathcal{E}}, \iota\right)$, the Abel parametrization of the Prym theta divisor $\Xi\left(\tilde{C}_{\mathcal{E}}, \iota\right)$, but $\mathcal{E}$ is in fact one of the 16 normalized push downs of Poincaré line bundles associated to $X\left(\tilde{C}_{\mathcal{E}}, \iota\right)$ above.

\section{Recovering hyperelliptic double covers with the same Prym variety as $\pi: \tilde{C} \rightarrow C$, from $X$.}

Next we show that $X$ determines geometrically a family of hyperelliptic double covers $\tilde{C}_{\lambda} / C_{\lambda}$ parametrized by the dual projective plane $\left|K_{C}\right|^{*}$, and whose Prym varieties are all isomorphic to $P(\tilde{C} / C)$. These hyperelliptic double covers occur as fibers of the map $h$ over lines $H_{\lambda}$ in $\left|K_{C}\right|$. We know from our calculation at the end of Section 2 that these curves $h^{-1}\left(H_{\lambda}\right)=\tilde{C}_{\lambda}$ have genus 5 . If $H$ is transverse to the branch curve $C^{*}$ then $\tilde{C}_{\lambda}$ is smooth, and we claim the involution on $X$ restricts to a fix point free one on $\tilde{C}_{\lambda}$. Indeed, if $\bar{p}+\bar{q}+\bar{r}+\bar{s}$ is a canonical divisor on $C$ without multiple points, then the $\pi$ inverse image on $\tilde{C}$ consists of eight distinct points $p+p^{\prime}+q+q^{\prime}+$ $r+r^{\prime}+s+s^{\prime}$ on $\tilde{C}$, so the involution $\iota$ on $X$ takes $(p+q+r+s)$ for instance to $p^{\prime}+q^{\prime}+r^{\prime}+s^{\prime}$. In general for any $D$ lying above $\bar{p}+\bar{q}+\bar{r}+\bar{s}, D$ and $\iota(D)$ have disjoint supports, and in particular $\iota$ has no fixed points on $X$ lying above $\bar{p}+\bar{q}+\bar{r}+\bar{s}$. Even if $D=2 \bar{p}+\bar{r}+\bar{s}$ is a general canonical divisor with multiple points, corresponding to a smooth point of $C^{*}, \iota$ does not fix any of the 6 points of $X$ lying over $D$. Indeed the only way we can have $\iota(D)=D$, is for $D$ to equal the union of two fibers of $\pi$, i.e. $D=p+p^{\prime}+q+q^{\prime}$. This occurs only over a canonical divisor of form $D=2 \bar{p}+2 \bar{q}$ on $C$, i.e. a divisor cut by one of the 28 bitangents to the canonical plane quartic $C$. Hence $\iota$ fixes at most 28 points of $X$, one over each node of the dual curve $C^{*}$. Thus if $H_{\lambda}$ is a line in $\left|K_{C}\right|$ transverse to $C^{*}$, in particular not containing a node or cusp of $C^{*}$, then $\tilde{C}_{\lambda}$ is smooth and $\iota$ restricts to a fix point free involution of $\tilde{C}_{\lambda}$. We claim next that $\varphi$ restricts on each such $\tilde{C}_{\lambda}$ to the "Abel-Prym" $\operatorname{map} \varphi: \tilde{C}_{\lambda} \rightarrow \Xi \subset P$.

To compute the degree of $\varphi$ on the curve $\tilde{C}_{\lambda}$ we must intersect $\tilde{C}_{\lambda}$ with a general $\mathbb{P}^{1}$ fiber of $\varphi$. Since the family $\left\{\tilde{C}_{\lambda}\right\}$ of inverse images of the lines $H_{\lambda}$ in $\left|K_{C}\right|$ is a linear system on the smooth variety $X$, that intersection 
number is constant over the linear system, and we have just computed it to be 2 at a reducible curve of the form $D_{p} \cup D_{p^{\prime}}$, the inverse image of a generic tangent line $H_{\bar{p}}$ to $C^{*}$. Thus $\varphi: \tilde{C}_{\lambda} \rightarrow \Xi \subset P$, is a degree two map from a smooth genus five curve with fix point free involution, to a two dimensional p.p.a.v., whose image is a one-cycle with homology class $2[\Xi]$. Moreover the involution induced on the source $\tilde{C}_{\lambda}$ by the involution on $X$, commutes with the natural involution $\mu \mapsto\left(K_{\tilde{C}}-\mu\right)$ on the target $\Xi \subset \operatorname{Pic}^{4}(\tilde{C})$. Hence, by Masiewicki's criterion [Ma], $\varphi$ is the Abel-Prym map from the curve $\tilde{C}_{\lambda}$ to $P$, and since $\varphi$ is 2:1 rather than injective, Masiewicki also implies that $\tilde{C}_{\lambda}$ is hyperelliptic. (Note that a special fiber $D_{p} \cup D_{p^{\prime}}$ of $h$ over a general tangent line $H_{\bar{p}}$ to $C^{*}$ at a smooth point representing a canonical divisor $2 \bar{p}+\bar{r}+\bar{s}$ on $C$ with $\bar{p}, \bar{r}, \bar{s}$, distinct, is a hyperelliptic "Wirtinger" double cover of a nodal curve with normalization isomorphic to $\Sigma \cong \Xi$, $\mathrm{cf}[\mathbf{B}$, p. 175 , Th. 5.4(i)].) This gives a parametrization of part of the hyperelliptic locus in the fiber of the Prym map over $(P, \Xi)$ by the projective plane $\left|K_{C}\right|^{*}$. Moreover, since the hyperelliptic Wirtinger covers obtained vary with one parameter, the smooth hyperelliptic covers vary with two parameters, hence give the generic smooth hyperelliptic double cover in the fiber $\mathcal{P}^{-1}(J(\Sigma), \Sigma)$.

\section{Remarks on some exceptional double covers.}

Following the notation of the introduction, if $\mathcal{M} / G$ is the moduli space of S-equivalence classes of semi-stable even $\mathbb{P}^{1}$ bundles over the smooth genus two curve $\Sigma$ with $\operatorname{Aut}(\Sigma) \cong \mathbb{Z}_{2}$, and $\mathcal{P}^{-1}(J(\Sigma))$ is the fiber of the Prym map over the Jacobian $J(\Sigma)$, we have shown that Yin's construction $(\tilde{C} \rightarrow$ $C) \mapsto X$ in $[\mathbf{Y}]$ provides an explicit inverse $\mathcal{P}^{-1}(J(\Sigma)) \rightarrow \mathcal{M} / G$ to the Narasimhan-Ramanan-Verra map, at least for those classical étale double covers $\pi: \tilde{C} \rightarrow C$ such that $\tilde{C}$ is smooth and non hyperelliptic. One can ask what the construction yields for other double covers in the fiber $\mathcal{P}^{-1}(J(\Sigma))$ of Beauville's extended Prym map $[\mathbf{B}]$, for instance those corresponding to points on the two components of the exceptional divisor of the composition $\mathcal{P}^{-1}(J(\Sigma)) \cong \sigma_{2}\left(\sigma_{1}(\mathcal{M} / G)\right)$ of the Narasimhan-Ramanan isomorphism with Verra's blowings up. General points of those two divisors correspond to étale double covers $\tilde{C} \rightarrow C$ where either $C$ and $\tilde{C}$ are smooth and hyperelliptic, or $C=\Sigma \cup E$ is an "elliptic tail" (a copy of $\Sigma$ joined at one point to an elliptic curve $E$ ) and $\tilde{C}=\Sigma_{1} \cup \tilde{E} \cup \Sigma_{2}$ is two copies of $\Sigma$ each joined at one point to an elliptic double cover $\tilde{E}$ of $E$. When $\tilde{C}$ is smooth and hyperelliptic, the Prym variety $P$ can still be realized as those line bundles $L$ in $\operatorname{Pic}^{4}(\tilde{C})$ with $\operatorname{Nm}(L)=\omega_{C}$ and $h^{0}(L)$ even, and thus we can define $X$ again by restricting the Abel map over $\Xi \subset P, \tilde{C}^{(4)} \supset X \rightarrow \Xi \subset P \subset \operatorname{Pic}^{4}(\tilde{C})$. In this case $X$ is always isomorphic to the trivial semi-stable $\mathbb{P}^{1}$ bundle $X \cong \Sigma \times\left|g_{2}^{1}(\tilde{C})\right| \cong \Sigma \times \mathbb{P}^{1}$, and hence $X$ cannot recover uniquely the hyperelliptic double cover $\tilde{C} \rightarrow C$. Interestingly, the restricted norm map 
$h: X \rightarrow\left|K_{C}\right|$ in this case is still finite and recovers at least the curve $C$ from the branch locus, as in Andreotti's proof of Torelli for hyperelliptic curves. The reason $h$ carries more information than $X$ here is that the net $h^{*}\left(\left|K_{C}\right|^{*}\right)$ is not complete on $X$; in fact $h^{0}\left(X, \mathcal{T}_{\varphi} \otimes \varphi^{*}\left(K_{\Sigma}\right)\right)=6$, so the map $h$ is not determined by $X$ in this case.

In the case where $C$ has an elliptic tail, even the definition of the generalized Prym variety as a subvariety of $\operatorname{Pic}^{4}(\tilde{C})$ breaks down. I.e. Beauville realizes the generalized Prym variety naturally in $\operatorname{Pic}^{2 g-2}(\tilde{C})$ (where $2 g-1=$ $\operatorname{genus}(\tilde{C}))$, only when the double cover satisfies condition $(*),([\mathbf{B}$, p. 157]). When $(*)$ holds, the multidegree $\underline{d}=\operatorname{deg}\left(\omega_{C}\right)$ is even, and $P$ is then defined naturally in $\operatorname{Pic}^{\underline{d} / 2}(\tilde{C})$, (Props. (3.10), (3.11) p. 162, and Rmk.(3.12) p. 163, which applies only to $(*)$ covers $)$. When $C=\Sigma \cup E$, then $\operatorname{deg}\left(\omega_{C}\right)=(3,1)$, $\operatorname{deg}\left(\omega_{\tilde{C}}\right)=(3,2,3)$, the multidegree is no longer even, in particular $(*)$ $\overline{\text { fails, }}$, there is no natural component of the reducible variety $\operatorname{Pic}^{4}(\tilde{C})$ in which to locate the base curve $\Sigma$ of the $\mathbb{P}^{1}$ bundle, and the construction given above of $X$ breaks down. When the double cover is an étale "Wirtinger cover" $\tilde{C}=\Sigma_{1} \cup \Sigma_{2} \rightarrow \Sigma / p \sim q=C$ ([V, 3.15, p. 442]), then $\underline{\operatorname{deg}}(\omega)=4, \underline{\operatorname{deg}}\left(\omega_{\tilde{C}}\right)=(4,4)$, hence evenness holds, and one can define a theta divisor $\tilde{\Theta}$ in $\operatorname{Pic}^{(2,2)}(\tilde{C})([\mathbf{B}], \S 2$ p. 153, Prop. (2.2) p. 155). Since this double cover is étale over the node however, $(*)$ is violated, and the Prym variety is apparently not realized in $\operatorname{Pic}^{(2,2)}(\tilde{C})$, i.e. Rmk. (3.12), p. 163, [B], seems to fail.

As a smooth étale double cover $\pi: \tilde{C} \rightarrow C$ becomes either an elliptic tail $\tilde{C}=\Sigma_{1} \cup \tilde{E} \cup \Sigma_{2} \rightarrow \Sigma \cup_{p} E=C$, or a "Wirtinger cover" $\tilde{C}=\Sigma_{1} \cup \Sigma_{2} \rightarrow$ $\Sigma / p \sim q=C$ (cf. [V], 3.15, 3.16, p. 442), (the two cases not satisfying Beauville's condition $(*)$ ), and hence $\operatorname{Pic}^{4}(\tilde{C})$ becomes reducible, it appears that the $\mathbb{P}^{1}$ bundles defined by the restricted Abel map $\tilde{C}^{(4)} \cup X \rightarrow \Xi \subset$ $P \subset \operatorname{Pic}^{4}(\tilde{C})$ become, in some of the components of $\operatorname{Pic}^{4}(\tilde{C})$, several copies of restrictions of the classical Abel map $\Sigma^{(3)} \rightarrow \operatorname{Pic}^{3}(\Sigma)$, restricted over Abel curves in $\operatorname{Pic}^{3}(\Sigma)$, i.e. $\Sigma^{(3)} \supset X \rightarrow \Sigma+p+q \subset \operatorname{Pic}^{3}(\Sigma)$ or $\Sigma^{(3)} \supset X \rightarrow$ $\Sigma+2 p \subset \operatorname{Pic}^{3}(\Sigma)$. If $p^{\prime}=\left|g_{2}^{1}-p\right|$, and $q^{\prime}=\left|g_{2}^{1}-q\right|$, then the curves $\Sigma / p \sim q \cong \Sigma / p^{\prime} \sim q^{\prime}$, and $\Sigma \cup_{p} E \cong \Sigma \cup_{p^{\prime}} E$ are isomorphic in pairs, but the bundles $\Sigma^{(3)} \supset X \rightarrow \Sigma+p+q \subset \operatorname{Pic}^{3}(\Sigma), \Sigma^{(3)} \supset X \rightarrow \Sigma+p^{\prime}+q^{\prime} \subset \operatorname{Pic}^{3}(\Sigma)$, $\Sigma^{(3)} \supset X \rightarrow \Sigma+2 p \subset \operatorname{Pic}^{3}(\Sigma)$, and $\Sigma^{(3)} \supset X \rightarrow \Sigma+2 p^{\prime} \subset \operatorname{Pic}^{3}(\Sigma)$ although semi-stable, are at least not obviously isomorphic. Hence depending on the model chosen for the nodal curve $C$, one would obtain more than one possible limiting $\mathbb{P}^{1}$ bundle. This seems to match up with the fact that a general $S$-equivalence class of a nonstable, semi-stable, even $\mathbb{P}^{1}$ bundle over $\Sigma$, has two nonsplit representative isomorphism classes which are interchanged by the hyperelliptic involution ([N-R], Rmk. 1, p. 35). Note that the definition of the bundles $X \rightarrow \Sigma+p+q$ and $X \rightarrow \Sigma+2 p$ uses the information of 
the marked points $p$, or $p$ and $q$ on the curves $C$, but not the $j$-invariant of the elliptic tail. The maps h appear to be defined by complete linear series, with branch loci dual to either the nodal quartic $\Sigma / p \sim q$ or the quartic model of $\Sigma$ with a cusp at $p$, so the $j$-invariant of the elliptic tail appears to be lost by the map $h$. A calculation shows that in each $S$-equivalence class corresponding to either a general elliptic tail or Wirtinger cover, there are exactly three nonisomorphic $\mathbb{P}^{1}$ bundles over $\Sigma$, one split and two nonsplit. One can construct a smooth, nonseparated moduli space containing both nonsplit bundles (see $[\mathbf{N}-\mathbf{R}]$ p. 21 , proof of Thm. 1), but there seems no reason to expect a natural construction which would pick out one of them. The split bundle is of course distinguished, but has too many deformations to be included naturally in a moduli space of isomorphism classes of general even $\mathbb{P}^{1}$ bundles over $\Sigma$, and we have not observed it arising even as a limit from the geometry of the Abel map construction in this paper.

\section{References}

[A] A. Andreotti, On a theorem of Torelli, Am. J. of Math., 80 (1958), 801-828.

[ACGH] E. Arbarello, M. Cornalba, P.A. Griffiths and J. Harris, Geometry of Algebraic Curves, Grund. der Math. Wissen, 267, Springer, (1985).

[A-M] A. Andreotti and A. Mayer, On period relations for abelian integrals on algebraic curves, Ann. Scuola Norm. Sup. Pisa, 21 (1967), 189-238.

[B] A. Beauville, Prym Varieties and the Schottky problem, Invent. Math., 41 (1977), 149-196.

[Ma] L. Masiewicki, Universal properties of Prym varieties with an application to algebraic curves of genus five, Trans. Am. Math. Soc., 222 (1976), 221-240.

[Mu1] D. Mumford, Prym Varieties I, in 'Contributions to Analysis', Academic Press, New York, (1974), 325-350.

[Mu2] - Theta characteristics of an algebraic curve, Ann. scient. Ec. Norm Sup., 4.4 (1971), 181-192.

[N-R] M.S. Narasimhan and S. Ramanan, Moduli of vector bundles on a compact Riemann surface, Annals of Math., 89(2) (1969), 14-51.

[V] A. Verra, The fibre of the Prym map in genus three, Math. Annalen, 276 (1987), 433-448.

[W] G. Welters, Abel-Jacobi Isogenies for Certain Types of Fano Threefolds, Mathematical Centre Tracts, Amsterdam, 141 (1981).

[Y] H. Yin, Deformation of special subvarieties of divisors associated to double covers of genus three curves, PhD thesis, University of Georgia, 1996.

Received July 21, 1997 and revised March 31, 1998.

University of GeORGiA

Athens, Georgia 30602

E-mail address: roy@math.uga.edu

University of GeORGiA

Athens, Georgia 30602 
E-mail address: rvarley@math.uga.edu 